\title{
Research
}

\section{Chakras' Energies Alterations in Patients with Chronic Gastritis}

\author{
Huang $\mathrm{WL}^{1 *}$, and Lopes $\mathrm{MH}^{2}$ \\ ${ }^{1}$ General Practitioner, Acupuncturist and Pain Specialist, Medical Acupuncture and Pain Management Clinic, Franca, Sao Paulo, Brazil \\ ${ }^{2}$ Fourth year medical student at Itajubá Medical School, Minas Gerais, Brazil
}

\begin{abstract}
${ }^{*}$ Correspondence to: Huang Wei Ling; Rua Homero Pacheco Alves, 1929, Franca, Sao Paulo, 14400-010, Brazil; Tel: 551637212437; Fax: 551637028540; E-mail: weilingmg@gmail.com
\end{abstract}

Received: Nov 11 ${ }^{\text {th }}, 2020$; Accepted: Dec $1^{\text {st }}, 2020$; Published: Dec $8^{\text {th }}, 2020$

Citation: Huang WL, Lopes MH. Chakras' energies alterations in patients with chronic gastritis. Gastro Open A Open J. 2020; I(1): 20-25. doi: 10.33169/gastro.GOAOJ-I-105

\begin{abstract}
Introduction

Gastritis is a general term for inflammation of the stomach. In traditional Chinese medicine (TCM), gastritis is a disharmony between Yin, Yang, $Q i$, Blood and Heat retention of Liver, Stomach, Spleen or other disharmonies.
\end{abstract}

\section{Purpose}

To demonstrate that gastritis has energies alterations and chakras' energies deficiencies as the root of its problem. The correction and replenishment of the chakras' energies meridians are the most important goals in patient's recovery.

\section{Methods}

Two case reports. The first, a 34-year-old male patient with complaints of chronic fatigue and epigastric pain, not improving with any medication the doctors prescribed. The second patient was a 32-year-old female who started having pain on the stomach and a burning sensation, as well as strong headache since she was 16-years-old. She was diagnosed with reflux through an endoscopy and treatment was started lansoprazole and pantoprazole and diet for reflux. She performed this treatment for four years, with no success. Both patients did the chakras' energies meridian measurement through radiesthesia procedure. The result was that six out of seven chakras' were completely lack of energies rated one (the minimum level) out of eight (the normal level), with the exception of the seventh chakra that was normal. Both patients were treated with Chinese dietary counseling, auricular acupuncture with apex ear bloodletting, systemic acupuncture and replenishment of the chakras' energies with homeopathic medication according to Constitutional Homeopathy of the Five Elements Based on Traditional Chinese Medicine and crystal based medications.

\section{Results}

Both patients recover from all their symptoms very fast, including the epygastric pain discomfort without the necessity of using any antacid and proton-pump inhibitors medications.

\section{Conclusion}

Gastritis has an energy alteration and chakras' energies deficiencies as a root and the treatment of these energy imbalances and replenishment of this lack of energies is essential for the patient's improvement in the deepest level, not only treating the symptom.

Keywords: Gastritis; Energy; Chakra; Traditional Chinese Medicine; Homeopathy; Crystal-based medication. 


\section{INTRODUCTION}

Gastritis is defined as an inflammation, irritation, or erosion of the lining of the stomach. It can occur suddenly (acute) or gradually (chronic). In Western medicine, gastritis can be caused by irritation due to excessive alcohol use, chronic vomiting, stress, or the use of certain medications such as aspirin or other anti- inflammatory drugs.

According to Mariya Varbanova, chronic gastritis may also be caused by Helicobacter pylori (H. pylori), a bacterium that lives in the mucous lining of the stomach. If lead without treatment, the bacteria can cause gastric ulcers, and in some people, stomach cancer. It can also be caused by bile reflux that is a backflow of bile into the stomach from the bile tract (that connects the liver and gallbladder). Infections caused by bacteria and viruses can lead to gastritis as well. ${ }^{2}$

If gastritis is left untreated, it can lead to a severe loss of blood and may increase the risk of developing stomach cancer. ${ }^{1,2}$

Symptoms of gastritis vary among individuals, and in many people there are no symptoms. However, the most common symptoms include nausea or recurrent upset stomach, abdominal bloating, abdominal pain, vomiting, indigestion, burning or gnawing feeling in the stomach between meals or at night, hiccups, loss of appetite, vomiting blood or coffee ground-like material, black stools. ${ }^{1,2}$

To diagnose gastritis, the doctor will perform a physical evaluation, and may recommend any of the following tests: upper endoscopy, blood tests, fecal occult blood test (stool test). ${ }^{1,2}$

Treatment of gastritis depends on the specific cause. Acute gastritis caused by nonsteroidal anti-inflammatory drugs or alcohol may be relieved by stopping use of those substances. ${ }^{1,2}$

In case of chronic gastritis, some of the medications used are: antibiotic medications to eliminate $\mathrm{H}$. pylori; medications that block acid production and promote healing; medications to reduce acid production; antacids that neutralize stomach acid. ${ }^{1,2}$

Traditional Chinese medicine (TCM) has accumulated many years of clinical experience in the diagnosis and treatment of this disease. The main diagnosis associated with gastritis is Liver- Stomach disharmony, Liver- Stomach Qi stagnation or Liver- Stomach Heat retention.

\section{PURPOSE}

The purpose of this study is to demonstrate that patients with gastritis symptoms have a chakras' energies deficiencies mainly in the fifth chakra that is responsible for the digestive system.

\section{METHODS}

It was done extensive research in gastritis subject in Western and traditional Chinese medicine PubMed articles. Also, two case reports were described in which both had symptoms of gastritis diagnosed and confirmed by upper gastrointestinal endoscopy, and both were diagnosed with completely deficiency in the chakras' energies in chakra one to six, with the exception of the seventh chakra that was normal.

\section{Case I}

Patient named J.C.M, 34-year-old male patient with complaints of chronic fatigue and epigastric pain. He made medical appointments and exams with several doctors who did not arrive at a diagnosis and only gave medications for his complaints, not improving his condition. The patient worked many hours in front of a computer in a big enterprise, in a very stressful environment.

The patient looked for a treatment with ancient medical tools. On the first examination, he was diagnosed according to traditional Chinese medicine, and the diagnosis was Yin, Yang, Q $i$ and Blood deficiencies and Heat retention.

The patient did the chakras' energies meridians measurement through radiesthesia procedure. The result was that six out of seven chakras' were completely lack of energy rated one (the minimum level) out of eight (the normal level), with the exception of the seventh chakra that was normal.

Treatment was started with Chinese dietary counseling that consists of avoiding foods that would harm the Spleen-pancreas, such as dairy products, raw food, sweets and cold drinks specially water. It was also recommended that the patient avoided foods that would harm the Kidney energy, that were coffee, soda and matte tea, as well as food that could cause Heat retention, such as fried food, eggs, honey, coconut, chocolate, alcoholic beverages, melted cheese. ${ }^{4}$

He received auricular acupuncture, on the points described in Figure 1, and apex ear bloodletting, twice a week. ${ }^{5}$

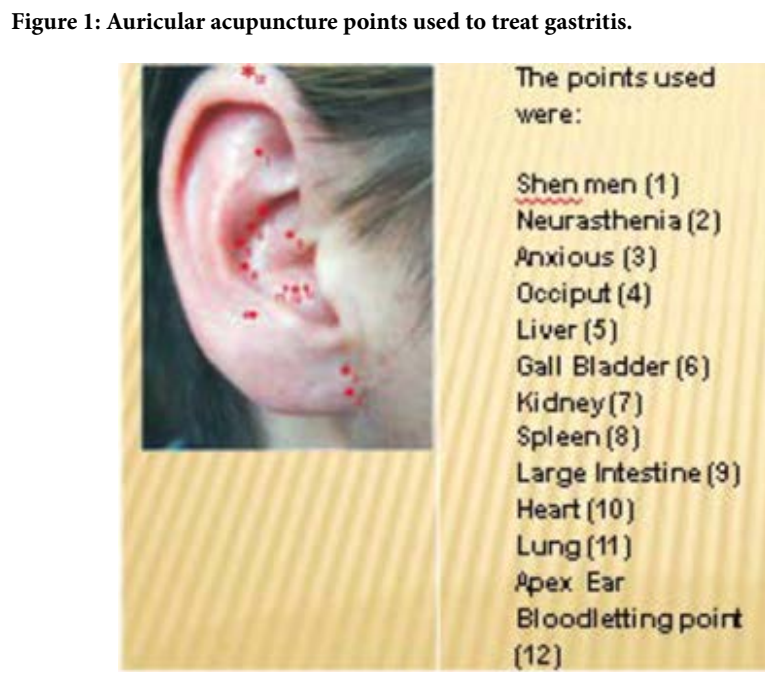

The patient received the recommendation of homeopathy during the period of one year or more, according to the theory created by the author Constitutional Homeopathy of the Five Elements Based on Traditional Chinese Medicine. The homeopathies medications used in the treatment were: Sulphur 30CHXX-20 (single dose), Calcarea carbonica 30CHXX-20 (single dose), Silicea 30CHXX-20 (single dose), Natrum muriaticum 30CHXX-20 (single dose), Phosphorus 30CHXX20. The homeopathies were prescribed to be taken in a single dose with a hiatus of three days between the medications, necessarily on the sequence described. Two months later, a new sequence of treatment was started, with the same medications taken on the same order, but now with 200CHXX-20 instead of 30CHXX-20. Two months later, a new sequence of treatment was started, as the others, with the same medications taken on the same order, now with 1,000CHXX-20 instead of 200CHXX-20.Two months later, a new sequence of treatment was started, as the others, with the same medications taken on the same or- 
der, now with 10,000CHXX-20 instead of 1,000CHXX-20.Two months later, a new sequence of treatment was started, as the others, with the same medications taken on the same order, now with 50,000CHXX-20 instead of 10,000CHXX-20. ${ }^{6}$

After one year of treatment it would be necessary to measure the energy of the chakras' again to assess the need to maintain or not the homeopathies medication treatment.

The crystal based medications prescribed will be described in the discussion section.

\section{Case 2}

Patient named, A.L.J., 32-year-old female patient. The background of the patient shown that when she was 16-years-old, she started having pain on the stomach and a burning sensation, as well as strong headaches. Three years later, she searched for a doctor and was diagnosed with the bacteria H-pylori (gastritis). Treatment was started with aluminum hydroxide, omeprazole, and antibiotics to treat the H-pylori bacteria.

The problem persisted, and she looked for a different doctor three years later. She was diagnosed with reflux through an endoscopy and treatment was started lansoprazole and pantoprazole and diet for reflux. She performed this treatment for four years, with no success. She performed surgery for reflux, maintaining the diet, lost 17 kilos in a month, had anemia, and lack of vitamin D.

She suffered with these symptoms for several years, having performing more than eight endoscopies, which would reveal gastritis, even though she did not respond well to the medications.

She then searched for treatment with ancient medical tools. She was first diagnosed with Yin, Yang, Qi and Blood deficiencies and Heat retention according to traditional Chinese medicine. She also received measurement of the chakras' energy meridians, and a deficiency was found in six of her seven chakras'. On a scale from 1 to 8 , six of her chakras' were rated in one, with the seventh chakra normal, in level $8{ }^{7}$

Treatment was started with Chinese dietary counseling, the same used in the case report one. She also received treatment with auricular acupuncture, apex ear bloodletting, and homeopathy according to the theory of Constitutional Homeopathy of the Five Elements Based on Traditional Chinese Medicine created by the author and crystal-based medications to replenish the energy of the chakras', the same prescribed in the first case report. ${ }^{6}$

\section{RESULTS}

\section{Results of Case I}

The patient had improvement of all his symptoms: lack of energy, fatigue, pour focus, hypertension, epigastric pain and muscular pain. His improvement was seen after each acupuncture session and the use of highly diluted medication (homeopathy and crystal based medication). He gained energy, feeling less tired, and being energized to work, improving his epigastric pain and normalizing his blood pressure.

\section{Results of Case 2}

The patient had great improvement of her symptoms, with the use of the Chinese dietary counseling, acupuncture associated with apex ear bloodletting, replenishing the lack of chakras' energy meridians with highly diluted medications (homeopathy and crystal based medication). With the treatment she was able to stop using the highly concentrated medications (aluminum hydroxide, omeprazole, and antibiotics) for the stomach that was prescribed in the beginning of her treatment that was not improving her symptoms.

\section{DISCUSSION}

To comprehend how the author did the reasoning of these two patients it is important to establish the metaphor of tree (Figure 2) that was used in many treatments and articles written by the author. Every study since 2006, when she first treated her first patient with this methodology, the author uses this treatment. ${ }^{4-6}$

Figure 2: Schematic drawing of the views of western and traditional Chinese medicine (Metaphor of the tree).

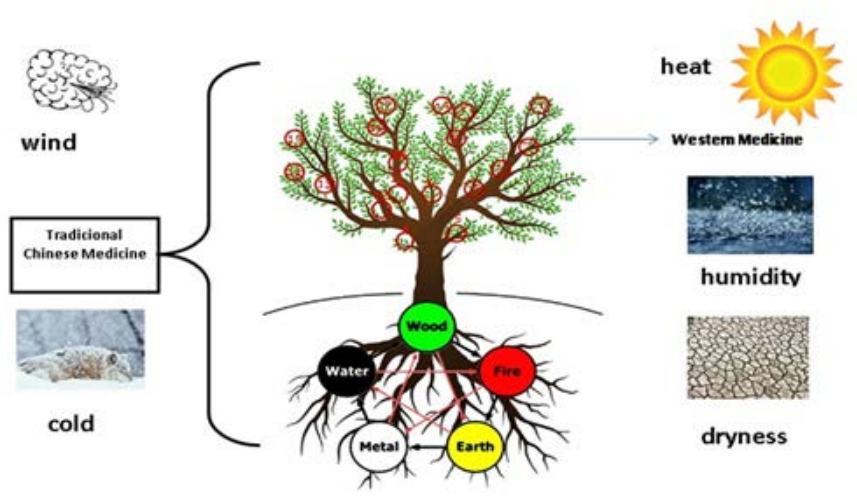

This patient in 2006, reported pain in the legs and was using anti-inflammatory medications without improvement and was diagnosed with Kidney-Yang deficiency, according to TCM. He received treatment with Chinese dietary counseling, acupuncture and auricular acupuncture associated with apex ear bloodletting. With the treatment done, the pain in the legs diminished and the patient was submitted to an interview after the treatment. In this interview, 30 days after the treatment, the patient revealed that his eye pressure had also lowered with the treatment, as his ophthalmologist confirmed. During the treatment, he had not reported to be treating glaucoma in the last 40 years with no improvement of his condition. With the treatment performed to treat the pain in the legs, his eye pressure lowers from $40 \mathrm{mmHg}$ to 17 mmHg. ${ }^{4-7}$

To demonstrate the different perspectives between Western medicine and traditional Chinese medicine, the author uses a metaphor of the tree. The tree has a trunk with several branches. Each branch represents one medical specialty and each leaf coming out of each branch represents the symptoms and diseases of each specialty. The focus is to treat each leaf in each specialty, in Western medicine. The treatment proposed by the author aims is to treat the entire tree, through the root to reach each leaf. ${ }^{4-7}$

In the root of the tree, there is energy that when is balanced can bring health to all parts. On Figure 2, it is possible to see how the roots of the tree are represented by the Yin and Yang theory and the Five Elements theory. On both cases described, it was able to see an imbalance of those energies (deficiencies of Yin, Yang, Qi, Blood and Heat retention) as demonstrated in the cases shown. ${ }^{4-7}$

The treatment in Western medicine is performed on the leaf 
level. In this study, it's proposed a deeper treatment, on the root level. The energies deficiencies found in both patients were leading to the formation of internal Heat that manifests in the stomach as the hyperemia found in the endoscopy exams..$^{4-7}$

In author daily practice, it is common for her to observe that patients diagnosed with gastritis have other digestive disorders such as gastroesophageal reflux. These problems could be associated with the deficiency of the fifth chakra, responsible for the Stomach, Spleen, Pancreas, thyroid and mammary glands in Ayurvedic medicine. ${ }^{10}$ In case report two the patient had reflux and did surgery to correct, but even after de surgery and taking medications for gastritis the symptoms did not disappeared. She only improved the symptoms after the treatment rebalancing the Yin, Yang, Qi, Blood and taking out Heat retention through Chinese dietary counseling, acupuncture associated with apex ear bloodletting and replenishing the chakras' energies meridians with highly diluted medications. ${ }^{4-8}$

In the article published by Christopher R. Chase, entitled The Geometry of Emotions: Using Chakra Acupuncture and 5-Phase Theory to Describe Personality Archetypes for Clinical Use, the author states that there is a correlation between the five emotions (fear, anger, joy, pensive thought, and grief) and the five elements (Water, Wood, Fire, Earth, and Metal) in traditional Chinese medicine and the treatment done in the chakras' energies meridians in Ayurvedic medicine.

In patients with gastritis, the most important chakra suffering from a deficiency is the fifth chakra. However, the author demonstrated in a study performed in her clinic in Brazil, which she measured through radiesthesia procedure the amount of energy found in a thousand patients in 2015 to 2020 period. From these patients, 409 patients' files aged from 2 to 80 years old were analyzed and the result was that more than $90 \%$ had a significant deficiency in all the chakras' which means that all internal massive organs representing the five elements (Liver, Heart, Spleen, Lung, Kidney) were in their lowest level, as you can see in the Figure 3. Each organ has its energy function. For example, the Kidney is responsible for the production of Yin and Yang energies. The Spleen is responsible for the absorption of nutrients and production of Blood and keeping the Blood inside the vessel. The Heart also commands the Blood flow inside the vessel. The Liver and the Lung are responsible for the distribution of the energy in the body. ${ }^{3}$

Figure 3: Five elements and the correspondent organ.

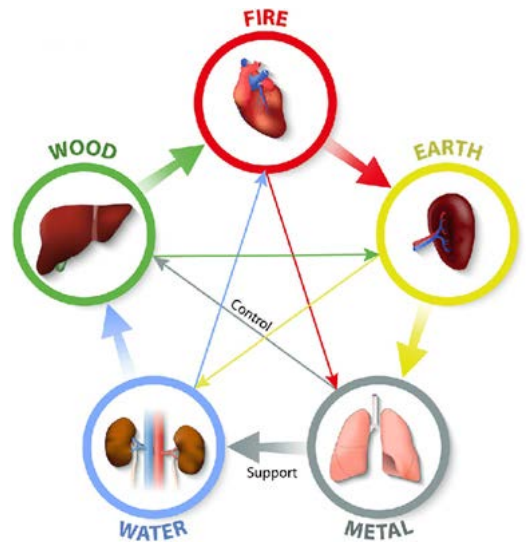

The author presented a study in 2015 in an Acupuncture Research Conference, held at Harvard medical school in Boston, USA. The study whose title was Acupuncture Viewed Holistically Can Treat All Patients Symptoms at the Same Time states that treating the energy imbalances between Yin, Yang, Q $i$ and Blood (Figure 4), the physician can treat all patient symptoms and diseases of all specialties at the same time even if the doctor does not know that the patient has that symptom or disease. This mean that, in her study of the chakras', where all organs are in the lowest level of energy, the production of Yin, Yang, Q $i$ and Blood energies are impaired to maintain the normal functioning of all organs and systems they command. ${ }^{9}$

Figure 4: Schematic relationship between Yin, Yang, $Q i$ and Blood.

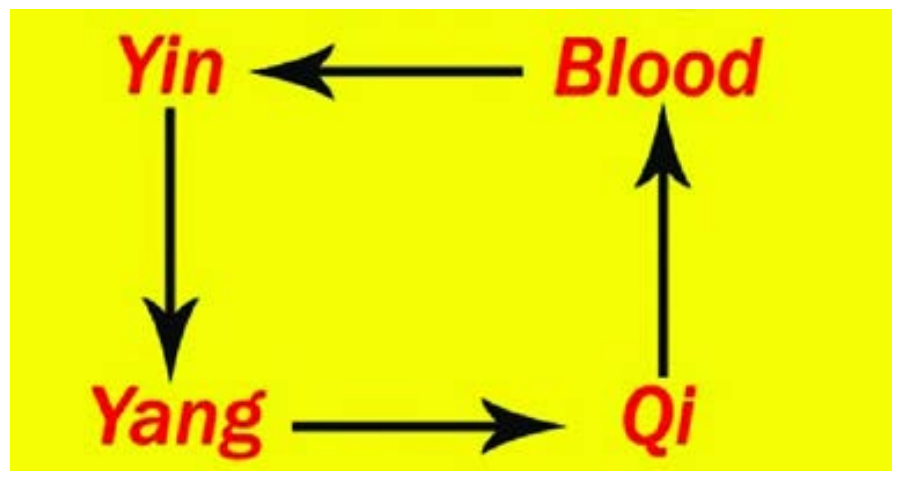

When there is energy deficiency in any of these energies, it can produce internal Heat formation, as you can see in Figure 5.

Figure 5: Energy deficiencies and formation of internal Heat.

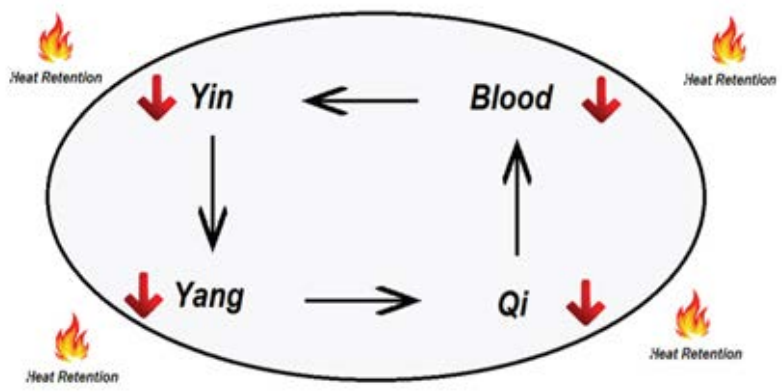

When performing an endoscopy in patients with gastritis, the stomach will appear red and inflamed. In traditional Chinese medicine, the inflammatory process is associated with Heat retention. As you can see on the Figure 5, deficiency from the massive organs or chakras' will lead to the formation of Heat. In both case reports presented in this study, all the chakras' were completely deficient of energy leading to the formation of internal Heat, in this case, it was leading to the gastritis manifestation (inflammatory process in the stomach due to lack of energy in the fifty chakra and subsequently formation of internal Heat). ${ }^{3-7}$

In Western medicine, the treatment is localized and focused on the stomach. The most common treatment is done with the use of antacid medications, such as omeprazole. ${ }^{10}$

The constant use of omeprazole may be associated with the development of cancer, as demonstrated on the study entitled The Safety of Long-term Daily Usage of a Proton Pump Inhibitor: A Literature Review. In the article, it is stated that literature demonstrates doubt regarding the safety in long term use of the medication, associating it with an increased risk 
of developing gastric cancer. ${ }^{11}$

The use of high-concentrated medications, such as antacids, would not be beneficial, because it would create a decrease in vital energy. According to the Arndt Schultz law, created in 1888 and illustrated in figure 6, medications in small doses stimulate, moderate doses inhibit and overdoses kill. In the case of the use high concentrated medication (proton pump inhibitor), it could induce more reduction in the vital energy that is already low, as demonstrated in both cases, inducing more internal Heat formation and this could be the reason why both patients did not improve their epigastric pain even using this kind of medication. ${ }^{8-12}$

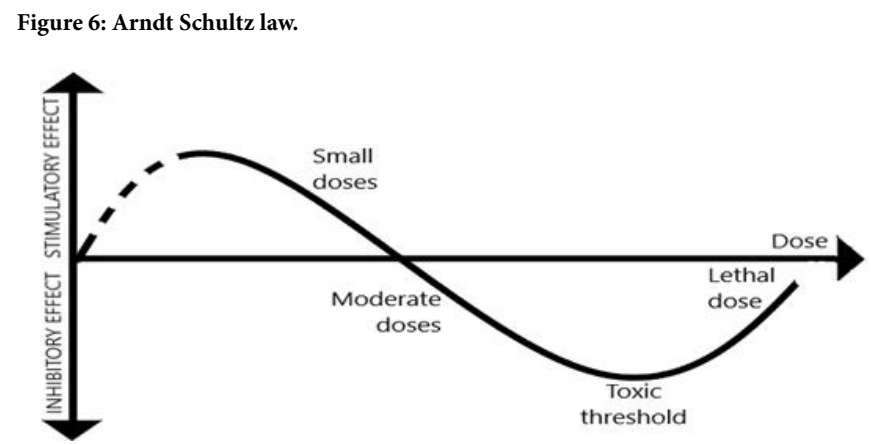

The non-treatment of this lack of energy may be a causative factor for the formation of cancer on the stomach on future, as the lack of energy and formation of internal Heat appears to be associated with the formation of cancer, according to TCM. ${ }^{13,14}$

Constant lack of energy may lead to different diseases, such as hypertension, myocardial infarction, diabetes, and even cancer, as described by the author in her previous studies also published in many journals. ${ }^{13-15}$

One of the study performed by the author, that supposed to be presented on Immunology and Infectious Diseases conference in Paris, but due to the corona virus pandemic, it was presented on a webinar on June $15^{\text {th }}, 2020$. In this study, its demonstrated that according to traditional Chinese medicine reasoning, cancer is formed by the lack of energy and the formation of Heat retention. It was also demonstrated that patients with cancer have deficiency of the chakras' energies and the treatment of the energies deficiencies is important for prevention of cancer, and either for its treatment, as demonstrated in two case reports, leading to a cure only using the rebalance of internal energies of Yin, Yang, Qi and Blood through Chinese dietary counseling, auricular acupuncture associated with apex ear bloodletting and replenishing the chakras' energies meridians using homeopathy medications according to the theory Constitutional Homeopathy of the Five Elements Based on Traditional Chinese Medicine, and crystal based medications. The descriptions of this last group of medications were well described in the article entitled Why are Diabetic Still Having Hyperglycemia Despite Diet Regulation, Antiglycemic Medication and Insulin. ${ }^{15,16}$

In order to understand how energies alterations develops gastritis, it is necessary to use other older medicines, as recommended by Hippocrates. ${ }^{17}$ The author emphasize that all the tools used in the treatment of the patient have been recognized by the Brazilian Medical Federal Council, such as acupuncture and homeopathy, since 1995 and $1980 .{ }^{18}$ The author creates the metaphor of the Yin and Yang, one repre- senting the Western medicine (Yin energy) that is materialized energy and traditional Chinese medicine represents Yang energy that is nonmaterialized energy and are indicated in Figure 7. Both medicines can work together to understand better how disease is formed in the deepest level, in the root of the tree represented in Figure 2, they are opposite but complementary. ${ }^{13-15}$

Figure 7: Traditional Chinese medicine and western medicine - Yin/Yang.

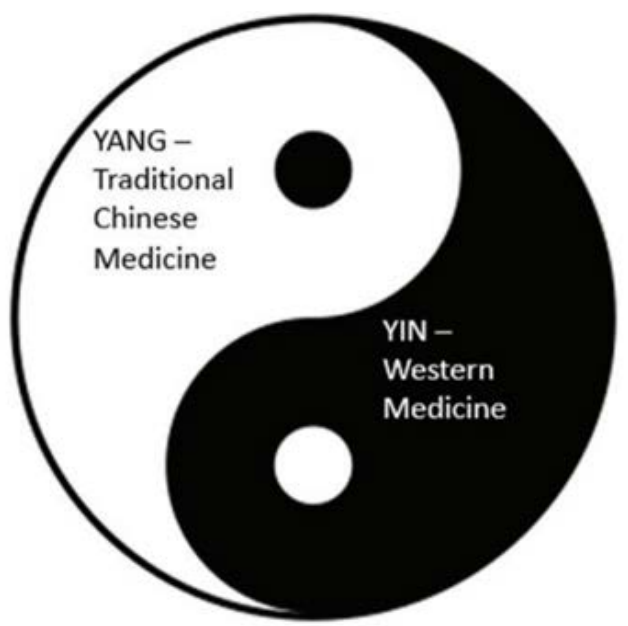

\section{CONCLUSION}

Patients with gastritis have lack of energy in the fifth chakra that is responsible for the digestive system, such as Stomach, Pancreas, Spleen, mammary gland, thyroid, and this lack of energy is leading to the formation of internal Heat, according to traditional Chinese medicine reasoning, and this internal Heat manifests as gastritis found in the endoscopy exams, shown as hyperemia on the stomach mucosa. The treatment of this lack of energy is important to reduce the formation of internal Heat, therefore reducing the inflammatory process in the stomach, leading to the reduction of the symptoms or cure in the deepest level of treatment.

\section{CONFLICTS OF INTEREST}

None.

\section{REFERENCES}

1. Sipponen P, Maaroos HI. Chronic gastritis. Scand J Gastroenterol. 2015; 50(6): 657-667. doi: 10.3109/00365521.2015.1019918

2. Varbanova M, Frauenschläger K, Malfertheiner P. Chronic gastritis - An update. Best Pract Res Clin Gastroenterol. 2014; 28(6): 1031-1042. doi: 10.1016/j.bpg.2014.10.005

3. Bing O, Zhen G. Essentials of traditional chinese medicine. Series of. Shandong Science and Technology Press; 1996; 209.

4. Huang WL. Auricular acupuncture and chinese dietary counselling in the treatment of insomnia. Arch Neurol Neuro Disord. 2020; 3(1): 1-11.

5. Huang WL. Can recurrent furunculosis be treated without the use of antibiotics? Acta Sci Microbiol. 2018; 1(9): 4-12.

6. Huang WL. Can We Treat Atopic Dermatitis Without Using Corticosteroids? Arch Gen Intern Med. 2018; 02(1): 8-19.

7. Huang WL. The Importance of Correcting Energy Imbalances and 
Chakras Energy Deficiencies in the Treatment of Patients with Glaucoma. Clinical Research in Ophthalmology. 2019.

8. LEESER O. Support of homeopathy by the Arndt-Schulz law. National Center for Biotechnology Information. 1953/ Jul 31.

9. Chase CR. The Geometry of Emotions: Using Chakra Acupuncture and 5-Phase Theory to Describe Personality Archetypes for Clinical Use. Med Acupunct. 2018; 30(4): 167-178.

10. Helgadottir H; Bjornsson E. Problems Associated with Deprescribing of Proton Pump Inhibitors. Int J Mol Sci. 2019; 20(21): 5469.

11. Abbas MK, Zaidi ARZ, Robert CA, Thiha S, Malik BH. The Safety of Long-term Daily Usage of a Proton Pump Inhibitor: A Literature Review. Cureus. 2019; 11(9)

12. Mastrangelo D. Hormesis, epitaxy, the structure of liquid water, and the science of homeopathy. Med Sci Monit. 2007; 13(1): 1-8.

13. Huang WL. Can Hospital Osteomyelitis Be Treated Without the Use of Antibiotics? Microbiol Infect Dis. 2018; 2(2):1-6

14. Huang WL. Why do Patients Still Catch Hospital Infections Despite the Practice of Infection Prevention and Control Programs? Acta Sci Microbiol. 2018; 1(4): 34-43.

15. Huang WL. Why Are Diabetic Patients Still Having Hyperglycemia despite Diet Regulation, Antiglycemic Medication and Insulin? Int $J$ Diabetes Metab Disord. 2019; 4(2): 1-14.

16. Huang WL. Constitutional Homeopathy of the Five Elements based on Traditional Chinese Medicine. Acta Sci Med Sci. 2020; 4(7): 57-69.

17. Yapijakis C. Hippocrates of Kos, the father of clinical medicine, and asclepiades of Bithynia, the father of molecular medicine. In Vivo (Brooklyn). 2009; 23(4): 507-514.

18. CREMEPE. HOMEOPATIA E ACUPUNTURA RECONHECIDAS E PROCURADAS. Novembro 2006. [Cited 2020 Dec 2]. Avaible from website: http://www.cremepe.org.br/2006/11/26/homeopatia-e-acupuntura-reconhecidas-e-procuradas. 